\title{
Inter-Observer Reliability of Physical Examination in the Painful Shoulder: Supraspinatus Tendinopathy
}

\author{
Jose Manuel Moreno-Fernandez ${ }^{*}$, Francisco Martinez-Martinez and Fernando Santonja-Medina \\ Orthopaedic Surgery and Traumatology Department, Hospital Clínico Universitario Virgen de la Arrixaca, Spain
}

\begin{abstract}
Objectives: Our hypothesis is that there is enough concordance in the implementation and interpretation of the orthopaedic maneuvers by expert explorers. The aim of our study was to analyze the inter-observer reliability of special orthopaedic maneuvers aimed at the physical examination of the supraspinatus tendon.

Setting: Secondary care; referral hospital for the Region de Murcia (Spain) fifth level of care.

Participants: 66 patients, 32 men and 34 women, were explored. The patients included were adults ( $\geq 18$ years), who suffered one-sided omalgia during at least 3 months.

Exclusion criteria were: Bilateral shoulder pain, fractures or previous dislocations, osteoarthritis and advanced retractable capsulitis, previous surgeries, less than 3 months of the last shoulder infiltration, cervical-brachialgias or neurological affectation, and the existence of obvious deficiency in the collaboration or understanding of the orders effected by the explorer.

Primary and secondary outcome measures: The physical assessment was conducted by two experienced explorers. The drop-arm test, the Jobe empty-can test, the full-can test and shrug sign, were carried out according to the original descriptions. Inter-observer concordance was studied.

Results: The highest levels of inter-explorer concordance were found in the drop-arm test (0.799 PABAK with an 84, $62 \%$ of agreement). The full-can test and shrug sign showed a good reliability, while the Jobe test presented a moderate reliability.

Conclusions: The drop-arm test, empty-can test, full-can test and shrug sign met the minimum criteria of percentage of agreement $>75 \%$ and 0.60 inter-observer reliability, by what were considered to be appropriate for their use in physical examination. Therefore, we consider that they are reproducible tests in medical practice for the diagnosis of the pathology of the supraspinatus tendon.
\end{abstract}

\section{Keywords}

Shoulder pain, Supraspinatus tendon, Physical examination, Inter-observer variation

\section{Article Summary}

\section{Highlights}

- It is important to have physical maneuvers with valid and reliable evidence.

- Drop-arm test inter-explorer reliability was the greatest, with a PABAK of 0.799 .

- Empty-can test inter-explorer reliability was moderate like published in literature.

- Full-can test inter-explorer reliability was good, without previous data in literature.

- Shrug sign had an acceptable inter-explorer reliability.

\section{Strengths and limitations of this study}

- The strength of the study is the strict adherence to a standardised study protocol for reproducibility studies, with a training and an overall agreement phase. We selected patients with painful shoulder, therefore this study will be applicable in medical practice.

*Corresponding author: José Manuel Moreno-Fernandez, Orthopaedic Surgery and Traumatology Department, Hospital Clínico Universitario Virgen de la Arrixaca, Ctra, Madrid-Cartagena, s/n, 30120, El Palmar, Murcia, Spain, Tel: +34-645489502

Accepted: March 11, 2019

Published online: March 13, 2019

Citation: Moreno-Fernandez JM, Martinez-Martinez F, Santonja-Medina F (2019) Inter-Observer Reliability of Physical Examination in the Painful Shoulder: Supraspinatus Tendinopathy. Arch Sports Med 3(1):129-133 
Citation: Moreno-Fernandez JM, Martinez-Martinez F, Santonja-Medina F (2019) Inter-Observer Reliability of Physical Examination in the Painful Shoulder: Supraspinatus Tendinopathy. Arch Sports Med 3(1):129-133

Table 1: Orthopaedic special maneuvers for the exploration used in the study of the rotator cuff.

\begin{tabular}{|l|l|l|}
\hline Test & Reference & Interpretation \\
\hline Drop arm test & {$[8]$} & $\begin{array}{l}\text { The appearance of pain or claudication of the affected limb } \\
\text { was considered as positive }\end{array}$ \\
\hline Jobe test or empty can test & {$[9]$} & $\begin{array}{l}\text { It was positive if pain or weakness appeared with respect to } \\
\text { the contralateral limb }\end{array}$ \\
\hline Jobe test in external rotation or full can test & {$[10]$} & The occurrence of pain was considered positive \\
\hline Shrug sign & {$[11]$} & $\begin{array}{l}\text { Considered positive in the elevation of the shoulder when } \\
\text { performing arm abduction }\end{array}$ \\
\hline
\end{tabular}

- The limitations could be related to measurement data from physical examination, the difference between observers, health care pressure during exploration, the cumulative effect of pain during the exploration, the existence of a washout period and the effect of rest pain.

\section{Introduction}

Shoulder pain is a significant cause of morbidity in the general population. In the United Kingdom it is estimated that its prevalence is $16 \%$ [1], increasing to $21 \%$ [2] in the 70 -yearold population. Each year, about $1 \%$ of adults over 45 -years in the United Kingdom presented a new episode of shoulder pain, of which only 40-50\% [3] were consulted for this reason, presenting an incidence of 15 new cases per year for every 1000 patients seen in primary care [4]. Painful shoulder is the third cause of inquiry due to skeletal muscle problems in primary care and the second cause of referral for specialist consultation $[1,5]$.

It is important to have physical maneuvers with valid and reliable evidence $[6,7]$ that complement a correct anamnesis, to obtain an accurate presumption diagnosis. Some orthopaedic maneuvers try to diagnose the affectation of the rotator cuff of the shoulder through pain provocation maneuvering. These tests can be the drop-arm test [8], the Jobe empty-can test [9], the full-can test [10] and the shrug sign [11]. The validity [12] and reliability [13-17] of these tests has been demonstrated.

Our hypothesis is that there is enough concordance in the implementation and interpretation of the orthopaedic maneuvers by expert explorers for the supraspinatus tendinopathy.

The aim of our study was to analyze the inter-observer reliability of maneuvers aimed at the physical exploration of the painful shoulder due to supraspinatus tendon pathology.

\section{Material and Methods}

\section{Selection of expert explorers}

The physical examination of patients was conducted by two Orthopaedic Surgery and Traumatology specialists with over 20 years of experience.

Both received at least 4 training sessions specific to the selected tests. Healthy volunteers were used in a first session to agree on the criteria of execution and interpretation of tests. In subsequent quarterly sessions suggestions and issues that arose were exposed and modifications and necessary clarifications were provided.

\section{Selection of patients}

The patients studied were treated from January 2013 to May 2014 by explorers in the consultations of Orthopaedic Surgery and Traumatology of the Hospital Clinico Universitario Virgen de la Arrixaca (Murcia, Spain), being included in the study consecutively according to the criteria laid down.

The patients included were men and women, adults ( $\geq$ 18 years), who suffered one-sided omalgia during at least 3 months.

Exclusion criteria were: Patients with bilateral shoulder pain, fractures, and previous dislocations that could alter the dynamics of the shoulder (middle or distal third of proximal humerus, clavicle and scapula), osteoarthritis and advanced retractable capsulitis, previous surgeries with the last shoulder infiltration of less than 3 months, symptoms of cervical brachialgias or neurological affectation, and the existence of obvious deficiency in the collaboration or understanding of the orders effected by the explorer.

\section{Evaluation of the shoulder}

We selected physical tests which have been proposed as more useful and reproducible in daily clinical practice, aimed at the assessment of the painful shoulder due to tendon pathology of supraspinatus (Table 1 ).

\section{Statistical analysis}

For the statistical calculations the Statistical Package for the Social Sciences (SPSS) version 19.0 (IBM Company) was used.

We calculated the concordance between observers of the tests and physical signs were assessed. For the qualitative variables the percentage of accord between the explorers and the corrected kappa coefficient [18]. We calculated the adjusted kappa index or PABAK (prevalence and bias adjusted kappa) [19] to take into account the degree of disaccord and differences between the proportions of positive and negative outcome that negatively affect the kappa coefficient [20-22].

To find out what were the limits of prevalence or bias affecting the overall kappa value, we calculated the rate of prevalence (PI) and bias (BI) for each variable index [19]. When the PI was high, we used the PABAK value to interpret the results of reliability. For our study, we determined a value of arbitrary cut of less than $-0.5 \mathrm{PI}$, or greater than 
Citation: Moreno-Fernandez JM, Martinez-Martinez F, Santonja-Medina F (2019) Inter-Observer Reliability of Physical Examination in the Painful Shoulder: Supraspinatus Tendinopathy. Arch Sports Med 3(1):129-133

0.5 , to base our interpretation on PABAK values instead of the kappa value. The kappa and PABAK values for the interexplorer reliability are interpreted in accordance with the recommendations of Landis and Kock [23]: Less than 0.20 poor; 0.21 to 0.40 regular; $0.41-0.60$, moderate; 0.61 to 0.80 , good; and 0.81 to 1 very good.

In our study, following the recommendations of Cadogan [17], we established minimum matching criteria so that a test may be considered appropriate for use in physical examination. When we used the absolute percentage of agreement, the minimum value that was established as acceptable was $75 \%[24,25]$. Regarding the inter-observer reliability, the minimum value that was accepted was 0.60 $[23,26]$.

\section{Results}

We evaluated 66 patients with shoulder pain, 32 men

Table 2: Descriptive data of the qualitative variables of the anamnesis.

\begin{tabular}{|l|l|l|}
\hline \multicolumn{1}{|l|}{} & $\mathbf{n}$ & $\%$ \\
\hline Sex & & \\
\hline Male & 32 & 48.48 \\
\hline Female & 34 & 51.51 \\
\hline Laterality & & \\
\hline Left & 17 & 25.76 \\
\hline Right & 49 & 74.24 \\
\hline Dominance & & \\
\hline Left & 4 & 6.06 \\
\hline Right & 62 & 93.94 \\
\hline
\end{tabular}

and 34 women, with an average age of 56 years (range 23-81 years). The average evolution time of painful symptoms was 13 months (Tables 2 and Table 3).

The values of $\mathrm{PI}, \mathrm{BI}$, corrected kappa, PABAK and the percentage of agreement with the special maneuvers of exploration are represented in Table 4. Inter-explorer concordance in this section is highly variable. The PI exceeded limits in all cases, so the PABAK values are representative of these tests. The PABAK values oscillated between 0.5050.799 and indicated moderate-good concordance between explorers. The percentage of agreement was $80 \%$ to $88 \%$.

\section{Discussion}

May [27] presented a systematic review of six studies of reliability on procedures of physical examination of painful shoulder, including 17 high quality studies, which showed conflicting results and most with values below acceptable levels of reliability. Nomden [14] presented a reliability study of 23 tests of shoulder girdle physical examinations made by physiotherapists. The tests were not standardized or backed by bibliography. They concluded that around $50 \%$ of the tests used did not meet the statistical criteria for acceptable reliability.

Cadogan [17] conducted a study with 40 patients and reported a good concordance between examiners (PABAK 0.67 ) in the drop-arm test. Our reliability in this case was greater, with a PABAK of 0.799 and a percentage of agreement of $84,62 \%$.

The empty-can test in our study showed a moderate reliability with a PABAK of 0.505. Ostor [13] studied 159 shoulders by a rheumatologist expert, a rheumatologist without experience in this field and a nurse, who were

Table 3: Descriptive data of the quantitative variables of the anamnesis.

\begin{tabular}{|c|c|c|c|c|c|}
\hline \multirow[b]{2}{*}{ Evolution time (months) } & \multirow{2}{*}{$\begin{array}{l}\text { Medium } \\
13\end{array}$} & \multicolumn{3}{|l|}{ Range } & \multirow[t]{2}{*}{$\mathbf{p}$} \\
\hline & & 3 & & 132 & \\
\hline Age (years) & 55.98 & 23 & - & 81 & \\
\hline - $\quad$ Males & 55.34 & 25 & - & 81 & \multirow[t]{2}{*}{0.732} \\
\hline - Females & 56.59 & 23 & - & 78 & \\
\hline DASH & 49.79 & & & & \\
\hline - $\quad$ Males & 45.48 & 12.5 & - & 81.67 & \multirow[t]{2}{*}{0.084} \\
\hline - $\quad$ Females & 54.10 & 19.16 & - & 89.17 & \\
\hline \multicolumn{6}{|l|}{ EVA } \\
\hline - $\quad$ Exploration 1 & 6.96 & & & & \multirow[t]{2}{*}{0.831} \\
\hline - $\quad$ Exploration 2 & 6.65 & & & & \\
\hline
\end{tabular}

Table 4: Reliability of special maneuvers of exploration data.

\begin{tabular}{|l|l|l|l|l|l|}
\hline Special maneuvers & \% agreement & PI & BI & Kappa corrected & PABAK \\
\hline Drop-arm test & 84.62 & 0.69 & 0.15 & 0.63 & 0.799 \\
\hline Jobe & 80.00 & 0.64 & 0.16 & 0.196 & 0.505 \\
\hline Jobe in ER & 88.00 & 0.76 & 0.12 & 0.452 & 0.761 \\
\hline Shrug sign & 80.00 & 0.60 & 0.20 & 0.611 & 0.735 \\
\hline
\end{tabular}


Citation: Moreno-Fernandez JM, Martinez-Martinez F, Santonja-Medina F (2019) Inter-Observer Reliability of Physical Examination in the Painful Shoulder: Supraspinatus Tendinopathy. Arch Sports Med 3(1):129-133

Table 5: Review of the reliability of special orthopaedic maneuvers of exploration of the shoulder

\begin{tabular}{|c|c|c|c|}
\hline Maneuvers & Reliability & Reference & Interpretation \\
\hline Drop-arm test & $\begin{array}{l}0.47(0.28-0.66) \\
0.467(0.275-0.659) \\
0.67 \\
\mathbf{0 . 7 9 9}\end{array}$ & $\begin{array}{l}{[30]} \\
{[13]} \\
{[17]} \\
\text { Our study }\end{array}$ & $\begin{array}{l}\text { Moderate } \\
\text { Moderate } \\
\text { Good } \\
\text { Good }\end{array}$ \\
\hline Test of Jobe & $\begin{array}{l}0.47(0.22-0.72) \\
0.46(0.44-0.49) \\
\mathbf{0 . 5 0 5}\end{array}$ & $\begin{array}{l}{[15]} \\
{[13]} \\
\text { Our study }\end{array}$ & $\begin{array}{l}\text { Moderate } \\
\text { Moderate } \\
\text { Moderate }\end{array}$ \\
\hline Test of Jobe in ER & $\begin{array}{l}\text { No previous study } \\
0.761\end{array}$ & - & - \\
\hline Shrug Sign & $\begin{array}{l}0.833 \\
\mathbf{0 . 7 3 5}\end{array}$ & $\begin{array}{l}{[29]} \\
\text { Our study }\end{array}$ & $\begin{array}{l}\text { Very good } \\
\text { Good }\end{array}$ \\
\hline
\end{tabular}

instructed by a phase of training. They presented interobserver moderate reliability for the Jobe test similar to our study, stressing the importance of sessions of updating and training of specialists for the diagnosis of painful shoulder management. Michener [15] studied 55 patients with painful shoulders that were explored by an orthopaedic surgeon and an expert physiotherapist using a combination of 5 physical tests for the diagnosis of subacromial impingement syndrome. For the test of Jobe, a moderate reliability, similar to our study, was shown.

Vind [16] studied shoulders in healthy athletes who were overstraining above their heads and reported a reliability of 0.9 with a percentage of agreement of $95 \%$. They included a training phase with 10 healthy volunteers, a phase of a global agreement with 20 players of handball and a phase study of $50 \%$ prevalence with 44 subjects, which may explain the greater concordance provided. Palmer [28] did not mention in his Protocol of Exploration of Southampton the Jobe test, classically described and used in our study, they presented it as the presence of pain in the shoulder to resisted abduction. The data presented in this study for this test were superior to ours, with a reliability of 0.81 and a percentage of agreement of $94 \%$.

The full-can test in our study showed good reliability with a PABAK 0.761 and a percentage of agreement of $88 \%$. We believe that this contribution is relevant, since we did not find data published in the literature consulted.

The shrug sign in our study showed a good reliability of 0.735 , with a percentage of agreement of $80 \%$. Jia [29] studied the inter-observer reliability of shrug sign, showing a very good reliability with a kappa of 0.833 .

The Table 5 shows a review of the reliability of special orthopaedic maneuvers used in our study.

We believe that there are several limitations that influenced this moderate-good overall reliability. These limitations could be related to measurement data from physical examination, the difference between observers, health care pressure during exploration, the cumulative effect of pain during the exploration, the existence of a washout period and the effect of rest pain.
Given that in the consulted literature we did not find any studies that analyze the reliability of the full-can test or set of tests of the supraspinatus, we believe that our work provides relevant information about the semiology of the supraspinatus tendon pathology.

\section{Conclusions}

The authors conclude that the drop-arm test, empty-can test, full-can test and shrug sign have an acceptable interexplorer reliability. Therefore, we consider that these tests are reproducible in clinical practice for the diagnosis of the pathology of the supraspinatus tendon.

\section{Acknowledgements}

The authors thank the selfless and voluntary participation of all patients included in this study.

\section{Contributorship Statement}

JMMF: Substantial contributions to the conception or design of the work; or the acquisition, analysis, or interpretation of data for the work; AND Drafting the work or revising it critically for important intellectual content; AND Agreement to be accountable for all aspects of the work in ensuring that questions related to the accuracy or integrity of any part of the work are appropriately investigated and resolved.

FMM: Substantial contributions to the conception or design of the work; or the acquisition, AND Final approval of the version to be published; AND Agreement to be accountable for all aspects of the work in ensuring that questions related to the accuracy or integrity of any part of the work are appropriately investigated and resolved.

FSM: Substantial contributions to the conception or design of the work; or the acquisition; AND Drafting the work or revising it critically for important intellectual content; AND Final approval of the version to be published; AND Agreement to be accountable for all aspects of the work in ensuring that questions related to the accuracy or integrity of any part of the work are appropriately investigated and resolved.

\section{Competing Interests}

All authors have completed the ICMJE uniform disclosure 
Citation: Moreno-Fernandez JM, Martinez-Martinez F, Santonja-Medina F (2019) Inter-Observer Reliability of Physical Examination in the Painful Shoulder: Supraspinatus Tendinopathy. Arch Sports Med 3(1):129-133

form at www.icmje.org/coi_disclosure.pdf and declare: No support from any organisation for the submitted work; no financial relationships with any organisations that might have an interest in the submitted work in the previous three years; no other relationships or activities that could appear to have influenced the submitted work.

\section{Funding}

There are no funders to report for this study.

\section{Data Sharing Statement}

No additional data available.

\section{References}

1. Urwin M, Symmons $D$, Allison T, et al. (1998) Estimating the burden of musculoskeletal disorders in the community: The comparative prevalence of symptoms at different anatomical sites, and the relation to social deprivation. Ann Rheum Dis 57: 649-655.

2. Chard MD, Hazleman R, Hazleman BL, et al. (1991) Shoulder disorders in the elderly: A community survey. Arthritis Rheum 34: 766-769.

3. Bongers PM (2001) The cost of shoulder pain at work. BMJ 322: 64-65.

4. Van der Windt DA, Koes BW, de Jong BA, et al. (1995) Shoulder disorders in general practice: Incidence, patient characteristics, and management. Ann Rheum Dis 54: 959-964.

5. Butcher JD, Zukowski CW, Brannen SJ, et al. (1996) Patient profile, referral sources, and consultant utilization in a primary care sports medicine clinic. J Fam Pract 43: 556-560.

6. Krebs DE (1987) Measurement theory. Phys Ther 67: 1834-1839.

7. Fritz JM, Wainner RS (2001) Examining diagnostic tests: An evidence-based perspective. Phys Ther 81: 1546-1564.

8. Codman EA (1934) The shoulder; rupture of the supraspinatus tendon and other lesions in or about the subacromial bursa, Boston.

9. Jobe FW, Jobe CM (1983) Painful athletic injuries of the shoulder. Clin Orthop Relat Res 173: 117-124.

10. Kelly BT, Kadrmas WR, Speer KP (1996) The manual muscle examination for rotator cuff strength. An electromyographic investigation. Am J Sports Med 24: 581-588.

11. Katrak PH (1990) Shoulder shrug--a prognostic sign for recovery of hand movement after stroke. Med J Aust 152: 297-301.

12. Hegedus EJ, Goode A, Campbell S, et al. (2008) Physical examination tests of the shoulder: A systematic review with meta-analysis of individual tests. Br J Sports Med 42: 80-92.

13. Ostor AJ, Richards CA, Prevost AT, et al. (2004) Interrater reproducibility of clinical tests for rotator cuff lesions. Ann Rheum Dis 63: 1288-1292.

14. Nomden JG, Slagers AJ, Bergman GJ, et al. (2009) Interobserver reliability of physical examination of shoulder girdle. Man Ther 14: 152-159.

15. Michener LA, Walsworth MK, Doukas WC, et al. (2009) Reliability and diagnostic accuracy of 5 physical examination tests and combination of tests for subacromial impingement. Arch Phys Med Rehabil 90: 1898-1903.

16. Vind $M$, Bogh SB, Larsen CM, et al. (2011) Inter-examiner reproducibility of clinical tests and criteria used to identify subacromial impingement syndrome. BMJ Open 1: e000042.

17. Cadogan A, Laslett M, Hing W, et al. (2011) Interexaminer reliability of orthopaedic special tests used in the assessment of shoulder pain. Man Ther 16: 131-135.

18. Cohen J (1960) A coefficient of agreement for nominal cales. Educ Psychol Meas 20: 37-46.

19. Byrt T, Bishop J, Carlin JB (1993) Bias, prevalence and kappa. J Clin Epidemiol 46: 423-429.

20. Feinstein AR, Cicchetti DV (1990) High agreement but low Kappa: I. The problems of two paradoxes. J Clin Epidemiol 43: 543-549.

21. Rigby AS (2000) Statistical methods in epidemiology. V. Towards an understanding of the kappa coefficient. Disabil Rehabil 22: 339-344.

22. Shankar V, Bangdiwala SI (2008) Behaviour of agreement measures in the presence of zero cells and biased marginal distributions. J Appl Statistics 35: 445-464.

23. Landis JR, Koch GG (1977) The measurement of observer agreement for categorical data. Biometrics 33: 159-174.

24. Hartmann DP (1977) Considerations in the choice of interobserver reliability measures. J Appl Behav Anal 10: 103-116.

25. Stemler SE (2004) A comparison of consensus, consistency, and measurement approaches to estimating interrater reliability. Practical Assessment, Research \& Evaluation 9: 1-11.

26. Altman DG (1991) Practical statistics for medical research, Champman \& Hall, London.

27. May S, Chance-Larsen K, Littlewood C, et al. (2010) Reliability of physical examination tests used in the assessment of patients with shoulder problems: A systematic review. Physiotherapy 96: 179-190.

28. Palmer K, Walker-Bone K, Linaker C, et al. (2000) The Southampton examination schedule for the diagnosis of musculoskeletal disorders of the upper limb. Ann Rheum Dis 59: 5-11.

29. Jia X, Ji JH, Petersen SA, et al. (2008) Clinical evaluation of the shoulder shrug sign. Clin Orthop Relat Res 466: 2813-2819.

30. Park HB, Yokota A, Gill HS, et al. (2005) Diagnostic accuracy of clinical tests for the different degrees of subacromial impingement syndrome. J Bone Joint Surg Am 87: 1446-1455.

DOI: $10.36959 / 987 / 240$

Copyright: @ 2019 Moreno-Fernandez JM, et al. This is an open-access article distributed under the terms of the Creative Commons Attribution License, which permits unrestricted use, distribution, and reproduction in any medium, provided the original author and source are credited. 